\title{
SOLID HULLS OF WEIGHTED BANACH SPACES OF ANALYTIC FUNCTIONS ON THE UNIT DISC WITH EXPONENTIAL WEIGHTS
}

\author{
José Bonet and Jari Taskinen \\ Universitat Politècnica de València, Instituto Universitario de Matemática Pura y Aplicada \\ IUMPA, E-46071 Valencia, Spain; jbonet@mat.upv.es \\ University of Helsinki, Department of Mathematics and Statistics \\ P.O. Box 68, 00014 Helsinki, Finland; jari.taskinen@helsinki.fi
}

\begin{abstract}
Let $v(r)=\exp \left(-a /(1-r)^{b}\right)$ with $a>0$ and $0<b \leq 2$ be an exponential weight on the unit disc. We study the solid hull of its associated weighted Banach space $H_{v}^{\infty}(\mathbf{D})$ of all the analytic functions $f$ on the unit disc such that $v|f|$ is bounded.
\end{abstract}

\section{Introduction}

Recently, the authors characterized in [7] the solid hulls of weighted $H^{\infty}$-type Banach spaces of entire functions for a large class of weight functions $v$. An analytic function $f(z)=\sum_{n=0}^{\infty} a_{n} z^{n}$ on the disc is identified with the sequence of its Taylor coefficients $\left(a_{n}\right)_{n=0}^{\infty}$. Let $A$ be a vector space of complex sequences containing the space of all the sequences with finitely many non-zero coordinates. The space $A$ is solid if $a=\left(a_{n}\right) \in A$ and $\left|b_{n}\right| \leq\left|a_{n}\right|$ for each $n$ implies $b=\left(b_{n}\right) \in A$. The solid hull of $A$ is

$$
S(A):=\left\{\left(c_{n}\right): \exists\left(a_{n}\right) \in A \text { such that }\left|c_{n}\right| \leq\left|a_{n}\right| \forall n \in \mathbf{N}\right\} .
$$

It coincides with the smallest solid space containing $A$. See [1].

The aim of this paper is to generalize the results of [7] for the corresponding spaces on the open unit disc D. Accordingly, we study Banach spaces $H_{v}^{\infty}(\mathbf{D})$ of analytic functions $f: \mathbf{D} \rightarrow \mathbf{C}$ such that $\|f\|_{v}:=\sup _{z \in \mathbf{D}} v(z)|f(z)|<\infty$. We show in Theorem 2.2 that the solid hull for $v(z)=\exp (-1 /(1-|z|)), z \in \mathbf{D}$, consists of complex sequences $\left(b_{m}\right)_{m=0}^{\infty}$ such that

$$
\sup _{n \in \mathbf{N}} \sum_{m=n^{4}+1}^{(n+1)^{4}}\left|b_{m}\right|^{2} \exp \left(-2 n^{2}\right)\left(1-\frac{1}{n^{2}}\right)^{2 m}<\infty .
$$

We also formulate a general Theorem 2.1, which contains the characterization of the solid hulls for a large class of weights. This class of weights includes those satisfying condition (B) of [13]. Theorem 2.2 is used to determine the space of multipliers from $H_{v}^{\infty}(\mathbf{D})$ into $\ell^{p}, 1 \leq p \leq \infty$, in Proposition 5.2.

Surprisingly enough, we will encounter a technical difficulty, which makes the calculation of the solid hulls for weights $v(z)=\exp \left(-a /(1-|z|)^{b}\right)$ on the disc more complicated than those for the somewhat analogous weights $v(z)=\exp \left(-a|z|^{b}\right)$ in the plane. The latter were successfully treated in [7] for all $b>0$ and $a>0$, but it seems to the authors that in the case of the disc the calculation of certain numerical sequences requires approximate solutions of some numerical equations, which can

https://doi.org/10.5186/aasfm.2018.4334

2010 Mathematics Subject Classification: Primary 46E15; Secondary 30H99, 46B45, 46 E05.

Key words: Weighted Banach spaces of analytic functions, solid hull, multipliers. 
only be done relatively simply for small enough $b$ : for the above mentioned weights, we only complete the calculation in the case $0<b \leq 2$.

Bennet, Stegenga and Timoney in their paper [2] determined the solid hull and the solid core of the weighted spaces $H_{v}^{\infty}(\mathbf{D})$ in the case the weight $v$ is doubling. Exponential weights $v(r)=\exp \left(-a /(1-r)^{b}\right)$ with $a, b>0$ are not doubling. Not much seems to be known about multipliers and solid hulls of weighted spaces of analytic functions on the unit disc in the case of exponential weights. Hadamard multipliers of certain weighted space $H_{a}^{1}(\alpha), \alpha>0$, were completely described by Dostanić in [9] (see also Chapter 13 in [12]). Other aspects of weighted spaces of analytic functions on the unit disc with exponential weights, like integration operators or Bergman projections, have been investigated recently by Constantin, Dostanić, Pau, Pavlović, Peláez and Rättyä, among others; see [8], [10], [14], [15] and [17]. The solid hull and multipliers on spaces of analytic functions on the disc has been investigated by many authors. In addition to [2], we mention for example [1], [11], the books [12] and [16] and the many references therein.

Spaces of type $H_{v}^{\infty}(\mathbf{C})$ and $H_{v}^{\infty}(\mathbf{D})$ appear in the study of growth conditions of analytic functions and have been investigated in various articles since the work of Shields and Williams, see e.g. [3], [4], [13], [18] and the references therein.

A weight $v$ is a continuous function $v:[0,1[\rightarrow] 0, \infty[$, which is non-increasing on $\left[0,1\right.$ [ and satisfies $\lim _{r \rightarrow 1} v(r)=0$. We extend $v$ to $\mathbf{D}$ by $v(z):=v(|z|)$. For such a weight, the weighted Banach space of analytic functions is denoted by $H_{v}^{\infty}(\mathbf{D})$ and its norm by $\|\cdot\|_{v}$. For an analytic function $f \in H(\mathbf{D})$, we denote $M(f, r):=$ $\max \{|f(z)||| z \mid=r\}$. Using the notation $O$ and $o$ of Landau, $f \in H_{v}^{\infty}(\mathbf{D})$ if and only if $M(f, r)=O(1 / v(r)), r \rightarrow 1$.

\section{The results}

As mentioned in the introduction, the solid hull of the weighted Banach space $H_{v}^{\infty}(\mathbf{D})$ of holomorphic functions on the unit disc $\mathbf{D}$ when the weight $v$ is doubling, i.e.

$$
\sup _{0<s \leq 1} \frac{v(1-s)}{v\left(1-2^{-1} s\right)}<\infty
$$

was determined in [2]. The doubling condition is equivalent to the condition $(*)$ of [13], see Example 2.4 of the citation. This condition appears also e.g. in [6, Theorem 3.2]. Examples of weights on the disc which are not doubling are given by $v(r):=\exp \left(-a /(1-r)^{b}\right), a>0, b>0$. In this section we investigate the solid hull of $H_{v}^{\infty}(\mathbf{D})$ for a large class of non doubling weights.

Given a continuous, radial weight $v$ and $m>0$, we denote by $r_{m}$ the global maximum point in $\left[0,1\right.$ [ of the function $r \mapsto r^{m} v(r)$. For $0<m<M$ we define

$$
A(m, M):=\left(\frac{r_{m}}{r_{M}}\right)^{m} \frac{v\left(r_{m}\right)}{v\left(r_{M}\right)} \text { and } B(m, M):=\left(\frac{r_{M}}{r_{m}}\right)^{M} \frac{v\left(r_{M}\right)}{v\left(r_{m}\right)} .
$$

A word by word repetition of the proof of [7, Theorem 2.5] yields the next general result; notice that the argument uses the results of [13], which are also established for holomorphic functions on the unit disc.

Theorem 2.1. Let $v$ be a radial weight on D. Let $0<m_{1}<m_{2}<\ldots$ be a sequence with $\lim _{n \rightarrow \infty} m_{n}=\infty$, such that for some constants $2<k \leq K$ we have

$$
k \leq A(n):=A\left(m_{n}, m_{n+1}\right) \leq K, \quad k \leq B(n):=B\left(m_{n}, m_{n+1}\right) \leq K
$$


for each $n \in \mathbf{N}$. Then, the solid hull of $H_{v}^{\infty}(\mathbf{D})$ is

$$
S\left(H_{v}^{\infty}(\mathbf{D})\right):=\left\{\left(b_{m}\right)_{m=0}^{\infty}: \sup _{n} v\left(r_{m_{n}}\right)\left(\sum_{m=m_{n}+1}^{m_{n+1}}\left|b_{m}\right|^{2} r_{m_{n}}^{2 m}\right)^{1 / 2}<\infty\right\} .
$$

Proceeding as in [7, Remark 2.7] one can show that every weight on the disc satisfying condition (B) of Lusky [13] satisfies the assumptions of Theorem 2.1; the definition of condition (B) is included at the end of this section.

The main result of this paper is the calculation of the solid hull for some of the most usual non-doubling weights on the unit disc, namely the weights

$$
v(r)=\exp \left(-a /(1-r)^{b}\right),
$$

where $a>0,0<b \leq 2$ are constants.

We write

$$
\alpha=2+\frac{2}{b}, \quad \beta=\frac{1}{1+b}, \quad G=(a b)^{\beta}
$$

and fix a positive number $S$ such that

$$
S \geq b a^{-1 / b} \alpha^{-1-1 / b}, \text { if } 0<b<2 \text {, and } S=2 a^{-1 / 2} \text {, if } b=2 .
$$

In particular (2.6) allows us to set $S=1$ in the special case $a=b=1$.

Theorem 2.2. If $0<b \leq 1$, then the solid hull $S\left(H_{v}^{\infty}(\mathbf{D})\right)$ of $H_{v}^{\infty}(\mathbf{D})$ consists of sequences $\left(b_{m}\right)_{m=0}^{\infty}$ satisfying

$$
\sup _{n \in \mathbf{N}} \exp \left(-2 a S^{b \beta} G^{-\beta} n^{2}\right) \sum_{m=S n^{2+2 / b}+1}^{S(n+1)^{2+2 / b}}\left|b_{m}\right|^{2}\left(1-\frac{G}{S^{\beta} n^{2 / b}}\right)^{2 m}<\infty .
$$

In the case $1<b \leq 2$, the condition for the solid hull is

$$
\begin{aligned}
& \sup _{n \in \mathbf{N}} \exp \left(-2 a G^{-\beta} S^{b \beta} n^{2}-2 \beta(a b)^{2 \beta} S^{(b-1) \beta} n^{2-2 / b}\right) \\
& \cdot \sum_{m=S n^{2+2 / b}+1}^{S(n+1)^{2+2 / b}}\left|b_{m}\right|^{2}\left(1-\frac{G}{S^{\beta} n^{2 / b}}+\frac{\beta G^{2}}{S^{2 \beta} n^{4 / b}}\right)^{2 m}<\infty .
\end{aligned}
$$

In particular, for $v(r)=\exp (-1 /(1-r))$ the solid hull is

$$
\left\{\left(b_{m}\right)_{m=0}^{\infty}: \sup _{n} \exp \left(-2 n^{2}\right) \sum_{m=n^{4}+1}^{(n+1)^{4}}\left|b_{m}\right|^{2}\left(1-\frac{1}{n^{2}}\right)^{2 m}<\infty\right\},
$$

(since $S=1$, see above), and for $v(r)=\exp \left(-1 /(1-r)^{2}\right)$ it is (!)

$$
\left\{\left(b_{m}\right)_{m=0}^{\infty}: \sup _{n \in \mathbf{N}} \exp \left(-2^{14 / 9} n^{2}-\frac{4}{3} n\right)_{m=(2 n)^{3}+1}^{2(n+1)^{3}}\left|b_{m}\right|^{2}\left(1-\frac{1}{n}+\frac{1}{3 n^{2}}\right)^{2 m}<\infty\right\}
$$

The proof requires the choice of the numbers $m_{n}$ such that the condition (2.2) is satisfied. This turns out to be quite technical and will be done in the next section. The main complication is that, contrary to the case of weights $\exp \left(-a r^{p}\right)$ of the paper [7], the maximum points $r_{m}$ cannot be solved explicitly (see (3.5)), and one has to treat only approximations of them (see Lemma 3.2) in the calculations. 
The proof of Theorem 2.2 will be completed in Section 4, where we also make some comments on the case $b>2$. Consequences of Theorem 2.2 for multipliers from $H_{v}^{\infty}(\mathbf{D})$ into $\ell_{p}, 1 \leq p \leq \infty$ are given in Section 5 .

At the end of this section we recall the condition (B) of Lusky [13], although we do not explicitly use it later: a weight $v$ having the general properties mentioned before (2.1) satisfies (B), if

$$
\begin{aligned}
& \forall b>1 \exists b_{2}>1 \exists c=c\left(b_{1}, b_{2}\right)>0 \forall m, n>0: \\
& \left(\frac{r_{m}}{r_{n}}\right)^{m} \frac{v\left(r_{m}\right)}{v\left(r_{n}\right)} \leq b_{1} \text { and } m, n,|m-n| \geq c \text { implies }\left(\frac{r_{n}}{r_{m}}\right)^{n} \frac{v\left(r_{n}\right)}{v\left(r_{m}\right)} \geq b_{2} .
\end{aligned}
$$

\section{Calculation of some numerical sequences}

For this section, let $v$ be as in (2.4). The next lemma will be crucial for the proof of Theorem 2.2, and its proof will occupy the whole section.

Lemma 3.1. For the weight $v$, the quantities $A(n)$ and $B(n)$ satisfy $(2.2)$, if $m_{n}$ is chosen to be

$$
m_{n}=S n^{2+\frac{2}{b}}=: S n^{\alpha},
$$

where $S=(16 a)^{-1}$ for $b=1, S=b a^{-1 / b} \alpha^{-1-1 / b}$ for $b<2$, and $S=2 a^{-1 / 2}$ for $b=2$.

We will need several times the Taylor expansions

$$
\begin{aligned}
(1+x)^{c} & =1+c x+\frac{1}{2} c(c-1) x^{2}+O\left(x^{3}\right), \\
\log (1+x) & =x-\frac{1}{2} x^{2}+\frac{1}{3} x^{3}+O\left(x^{4}\right),
\end{aligned}
$$

valid for $|x|<1$ and $c \in \mathbf{R}$. We start with the following estimate. Recall that $\alpha, \beta$ and $G$ are defined in (2.5).

Lemma 3.2. Given $m \geq \max \{1, a b\}$, the global maximum point $r_{m}$ of the function $r \mapsto r^{m} v(r)$ satisfies the estimate

$$
1-\frac{G}{m^{\beta}}+\frac{\beta G^{2}}{m^{2 \beta}}-\frac{C}{m^{3 \beta}} \leq r_{m} \leq 1-\frac{G}{m^{\beta}}+\frac{\beta G^{2}}{m^{2 \beta}}+\frac{C}{m^{3 \beta}} .
$$

Proof. A simple calculation shows that $r_{m}$ satisfies the equation

$$
m\left(1-r_{m}\right)^{b+1}-a b r_{m}=0 .
$$

It is obvious that for some fixed $0<\epsilon \leq 1 / 2$, the equation (3.5) has a solution $r_{m} \in\left[\epsilon, 1\left[\right.\right.$, for all $m \geq 1$. Writing $\left.\left.\delta=1-r_{m} \in\right] 0,1\right],(3.5)$ is equivalent with

$$
m^{\frac{1}{b+1}} \delta=(a b)^{\frac{1}{b+1}}(1-\delta)^{\frac{1}{b+1}}=(a b)^{\frac{1}{b+1}}\left(1-\frac{1}{b+1} \delta-\frac{b}{2(b+1)^{2}} \delta^{2}-+\ldots\right),
$$

or, by $(2.5)$,

$$
\delta=\frac{G}{m^{\beta}}\left(1-\beta \delta+\delta^{2} F(a, b, \delta)\right)
$$

where the expression $F(a, b, \delta)$ is for fixed $a, b$, uniformly bounded in $\delta \in] 0,1-\epsilon]$. (The choice of $\epsilon$ is needed here as $F$ would not be uniformly bounded for $\delta \in] 0,1]$.) We already noticed that (3.6) has a solution $\delta \in[0,1-\epsilon]$. Setting the estimate $\delta \leq 1$ to the right hand side of (3.6) yields the bound $\delta \leq \mathrm{Cm}^{-\beta}$. Since $m \geq a b$ 
by assumption, we have $G m^{-\beta} \leq 1$ and thus the solution $x$ ((3.9), below) of the equation

$$
x=\frac{G}{m^{\beta}}(1-\beta x)
$$

satisfies $0<x<1$; putting the bound $x \leq 1$ to the right hand side of (3.7) yields $x \leq G m^{-\beta}$. Then, subtracting (3.7) from (3.6) and using the triangle inequality,

$$
|\delta-x| \leq \frac{G \beta}{m^{\beta}}|\delta-x|+\frac{G \delta^{2}}{m^{\beta}}|F| .
$$

But we have $|x-\delta| \leq x+\delta \leq C m^{-\beta}$, and putting this to the right hand side of (3.8) implies $|\delta-x| \leq C m^{-2 \beta}$. Substituting this once more in (3.8) yields $|\delta-x| \leq C m^{-3 \beta}$. This implies (3.4), since the solution of (3.7) is

$$
x=\frac{G m^{-\beta}}{1+\beta G m^{-\beta}}=G m^{-\beta}-\beta G^{2} m^{-2 \beta}+O\left(m^{-3 \beta}\right) .
$$

From now on we assume that $m_{n}$ is defined for all $n \in \mathbf{N}$ as in (3.1). By (3.4) we can write for all $n$ such that $m_{n} \geq \max \{1, a b\}$,

$$
1-r_{m_{n}}=G m_{n}^{-\beta}-\beta G^{2} m_{n}^{-2 \beta}+R\left(m_{n}\right)
$$

where $\left|R\left(m_{n}\right)\right| \leq C m_{n}^{-3 \beta}$.

Lemma 3.3. Let $0<b \leq 2$. We have for all large enough $n \in \mathbf{N}$

$$
\log \left(\frac{v\left(r_{m_{n+1}}\right)}{v\left(r_{m_{n}}\right)}\right)= \begin{cases}-\frac{2 G}{b} S^{b \beta} n-\frac{G}{b} S^{b \beta}+O\left(n^{-1}+n^{1-2 / b}\right), & \text { if } b<2, \\ -G S^{2 / 3} n-\frac{G}{2} S^{2 / 3}-\frac{1}{3} G^{2} S^{1 / 3}+O\left(n^{-1}\right), & \text { if } b=2 .\end{cases}
$$

Notice that in the case $b=2$ we have $b \beta=2 / 3$ and more importantly, the constant term (of order $n^{0}$ ) is not the same as what would be gotten from the formula in the case $b<2$.

Proof. Using (3.10) and (3.2), and assuming that $n$ is so large that $G m_{n}^{-\beta}<1$, we get

$$
\begin{aligned}
v\left(r_{m_{n}}\right) & =\exp \left(-a\left(G m_{n}^{-\beta}-\beta G^{2} m_{n}^{-2 \beta}+R\left(m_{n}\right)\right)^{-b}\right) \\
& =\exp \left(-a G^{-b} m_{n}^{b \beta}\left(1-\beta G m_{n}^{-\beta}+G^{-1} m_{n}^{\beta} R\left(m_{n}\right)\right)^{-b}\right) \\
& =\exp \left(-a G^{-b} m_{n}^{b \beta}-a b \beta G^{1-b} m_{n}^{(b-1) \beta}+\tilde{R}_{n}\right)
\end{aligned}
$$

where $\left|\tilde{R}_{n}\right| \leq C m_{n}^{\beta(b-2)}$. We thus get, taking into account that $m_{n}=S n^{\alpha}$ and using (3.2) again,

$$
\begin{aligned}
\log \left(\frac{v\left(r_{m_{n+1}}\right)}{v\left(r_{m_{n}}\right)}\right)= & -a G^{-b}\left(m_{n+1}^{b \beta}-m_{n}^{b \beta}\right)-a b \beta G^{1-b}\left(m_{n+1}^{(b-1) \beta}-m_{n}^{(b-1) \beta}\right)+\tilde{R}_{n+1}-\tilde{R}_{n} \\
= & -a G^{-b} S^{b \beta}\left((n+1)^{b \alpha \beta}-n^{b \alpha \beta}\right) \\
& -a b \beta G^{1-b} S^{(b-1) \beta}\left((n+1)^{(b-1) \alpha \beta}-n^{(b-1) \alpha \beta}\right)+\tilde{R}_{n+1}-\tilde{R}_{n} \\
= & -a G^{-b} S^{b \beta} b \alpha \beta n^{b \alpha \beta-1}-a G^{-b} S^{b \beta} \frac{1}{2} b \alpha \beta(b \alpha \beta-1) n^{b \alpha \beta-2}+O\left(n^{b \alpha \beta-3}\right) \\
& -a b \beta G^{1-b} S^{(b-1) \beta}(b-1) \alpha \beta n^{(b-1) \alpha \beta-1}+O\left(n^{(b-1) \alpha \beta-2}\right) .
\end{aligned}
$$


This is simplified to the claimed form by observing that

$$
\begin{aligned}
a b G^{-b} & =(a b)^{1-\frac{b}{1+b}}=(a b)^{\beta}=G \\
\alpha \beta & =2(1+b) b^{-1}(b+1)^{-1}=2 / b, \quad b \alpha \beta=2
\end{aligned}
$$

and moreover

$$
(b-1) \alpha \beta-1=b \alpha \beta-1-\alpha \beta=1-2 b^{-1} \begin{cases}<0, & b<2, \\ =0, & b=2 .\end{cases}
$$

Lemma 3.4. If $0<b<2$, we have for all large enough $n \in \mathbf{N}$,

$$
m_{n+1} \log \left(\frac{r_{m_{n+1}}}{r_{m_{n}}}\right)=\frac{2 G}{b} S^{b \beta} n+\frac{G}{b}\left(3+\frac{2}{b}\right) S^{b \beta}+O\left(n^{-1}+n^{1-2 / b}\right) .
$$

If $b=2$, then

$$
m_{n+1} \log \left(\frac{r_{m_{n+1}}}{r_{m_{n}}}\right)=G S^{2 / 3} n+2 G S^{2 / 3}+\frac{1}{3} G^{2} S^{1 / 3}+O\left(n^{-1}\right) .
$$

Proof. We have again by (3.10), (3.3), for large enough $n$,

$$
\begin{aligned}
& m_{n+1} \log \left(\frac{r_{m_{n+1}}}{r_{m_{n}}}\right)=m_{n+1}\left(\log \left(1-G m_{n+1}^{-\beta}+\beta G^{2} m_{n+1}^{-2 \beta}+R\left(m_{n+1}\right)\right)\right. \\
& \left.\quad-\log \left(1-G m_{n}^{-\beta}+\beta G^{2} m_{n}^{-2 \beta}+R\left(m_{n}\right)\right)\right) \\
& =m_{n+1}\left(-G\left(m_{n+1}^{-\beta}-m_{n}^{-\beta}\right)+\left(\beta-\frac{1}{2}\right) G^{2}\left(m_{n+1}^{-2 \beta}-m_{n}^{-2 \beta}\right)+O\left(m_{n}^{-3 \beta}\right)\right) .
\end{aligned}
$$

Here, keeping in mind that $m_{n}=S n^{\alpha}$, the term

$$
m_{n+1}\left(\beta-\frac{1}{2}\right) G^{2}\left(m_{n+1}^{-2 \beta}-m_{n}^{-2 \beta}\right)
$$

is of degree $\alpha-2 \beta \alpha-1=1-2 / b$ with respect to $n$, and this number is negative, if and only if $b<2$. So, in the case $b<2$ we obtain using $1-\beta=b \beta$ and (3.2)

$$
\begin{aligned}
- & m_{n+1} G\left(m_{n+1}^{-\beta}-m_{n}^{-\beta}\right) \\
= & -S(n+1)^{\alpha} G S^{-\beta}\left(-\alpha \beta n^{-\alpha \beta-1}+\frac{1}{2} \alpha \beta(\alpha \beta+1) n^{-\alpha \beta-2}+O\left(n^{-\alpha \beta-3}\right)\right) \\
= & -G S^{b \beta}\left(n^{\alpha}+\alpha n^{\alpha-1}+O\left(n^{\alpha-2}\right)\right) \\
& \cdot\left(-\alpha \beta n^{-\alpha \beta-1}+\frac{1}{2} \alpha \beta(\alpha \beta+1) n^{-\alpha \beta-2}+O\left(n^{-\alpha \beta-3}\right)\right) .
\end{aligned}
$$

As for the exponents, notice that

$$
\alpha-\alpha \beta-1=2+2 b^{-1}-2 b^{-1}-1=1 .
$$

The coefficient of $n$, respectively, $n^{0}$, thus equals

$$
G S^{b \beta} \alpha \beta, \quad \text { resp. } \quad G S^{b \beta}\left(-\frac{1}{2} \alpha \beta(\alpha \beta+1)+\alpha^{2} \beta\right) .
$$

This yields the claim of the lemma for $b<2$ by using (3.14).

In the case $b=2$ the term (3.17) equals

$$
S(n+1)^{\alpha}\left(\beta-\frac{1}{2}\right) G^{2} S^{-2 \beta}\left(-2 \alpha \beta n^{-2 \alpha \beta-1}+O\left(n^{-2 \alpha \beta-2}\right)\right)=\frac{1}{3} S^{1 / 3} G^{2}+O\left(n^{-1}\right) .
$$


Adding this to the previous case yields (3.16).

Lemma 3.5. If $0<b \leq 2$, we have for all large enough $n \in \mathbf{N}$,

$$
m_{n} \log \left(\frac{r_{m_{n+1}}}{r_{m_{n}}}\right)= \begin{cases}\frac{2 G}{b} S^{b \beta} n-G S^{b \beta} \frac{1}{b}\left(\frac{2}{b}+1\right)+O\left(n^{1-2 / b}\right), & b<2, \\ G S^{2 / 3} n-G S^{2 / 3}+\frac{1}{3} S^{1 / 3} G^{2}+O\left(n^{-1}\right), & b=2 .\end{cases}
$$

Proof. One makes the obvious change $m_{n+1} \rightarrow m_{n}$, or, $(n+1)^{\alpha} \rightarrow n^{\alpha}$ in the proof of Lemma 3.4 and collects the coefficients of the remaining terms in the same way as in the argument (3.18) (one obtains (3.20) except for the term $\alpha^{2} \beta$ ). The case $b=2$ is proven in the same way: in addition to the omission of the $\alpha^{2} \beta$-term there are no other changes.

Proof of Lemma 3.1. Let first $b<2$. We consider the quantity

$$
\log B(n)=m_{n+1} \log \left(\frac{r_{m_{n+1}}}{r_{m_{n}}}\right)+\log \left(\frac{v\left(r_{m_{n+1}}\right)}{v\left(r_{m_{n}}\right)}\right)
$$

and first observe that the coefficients of the term with $n$ are the opposite numbers in (3.11) and (3.15). The sum of the coefficients of the term $n^{0}$ in (3.11) and (3.15) is

$$
G S^{b \beta}\left(-\frac{1}{b}+\frac{3}{b}+\frac{2}{b^{2}}\right)
$$

so that we get

$$
\log B(n)=G S^{b \beta}\left(\frac{2}{b}+\frac{2}{b^{2}}\right)+O\left(n^{-1}+n^{1-2 / b}\right)=G S^{b \beta} \frac{\alpha}{b}+O\left(n^{-1}+n^{1-2 / b}\right)
$$

The required property (2.2) follows for $B(n)$ by choosing $S$ large enough so that the constant term on the right of (3.22) is at least 1 . We choose $S$ such that

$$
G S^{b \beta} \geq \frac{b}{\alpha} \Longrightarrow S \geq b a^{-1 / b} \alpha^{-1-1 / b} .
$$

The same calculation, using Lemma 3.5 instead of Lemma 3.4, yields (notice the order of the numerator and denominator in $A(n)$ )

$$
\begin{aligned}
\log A(n) & =G S^{b \beta}\left(\frac{1}{b}+\frac{1}{b}\left(\frac{2}{b}+1\right)\right)+O\left(n^{-1}+n^{1-2 / b}\right) \\
& =G S^{b \beta} \frac{\alpha}{b}+O\left(n^{-1}+n^{1-2 / b}\right),
\end{aligned}
$$

and we get the desired conclusion for $A(n)$ by the same choice of $S$ as above.

Finally, if $b=2$, we have instead of (3.23)

$$
\log A(n)=\frac{3}{2} G S^{2 / 3}+O\left(n^{-1}\right)
$$

where $G=(2 a)^{1 / 3}$. Choosing

$$
S=2 a^{-1 / 2}
$$

the leading term in (3.24) is at least one, hence, (2.2) follows for $A(n)$, if $n$ is large enough.

We have instead of (3.22) the estimate (cf. (3.11) and (3.16))

$$
\log B(n)=\frac{3}{2} G S^{2 / 3}+O\left(n^{-1}\right)
$$

and we thus again see by $(3.25)$ that $\log B(n) \geq 1+O\left(n^{-1}\right)$. 


\section{Proof of Theorem 2.2.}

We choose $m_{n}$ according to (3.1) for all $n \in \mathbf{N}$. Theorem 2.2 follows in principle from Theorem 2.1 and Lemma 3.1, but we need to be careful to use accurate enough approximations of $r_{m_{n}}$. If $b \leq 1$, we observe that in (3.12), the exponent of $n^{(b-1) \alpha \beta}=$ $n^{2-2 / b}$ in $m_{n}^{(b-1) \beta}$ is at most 0 and $\left|\tilde{R}_{n}\right|$ is clearly bounded by a constant, so we get for all $n \in \mathbf{N}$ using (3.12) and (3.14),

$$
c_{1} \exp \left(-a G^{-b} S^{b \beta} n^{2}\right) \leq v\left(r_{m_{n}}\right) \leq c_{2} \exp \left(-a G^{-b} S^{b \beta} n^{2}\right),
$$

where

$$
\begin{aligned}
& c_{1}=\exp \left(\inf _{n}\left(-a b \beta G^{1-b} S^{(b-1) \beta} n^{2-2 / b}-\left|\tilde{R}_{n}\right|\right)\right), \\
& c_{2}=\exp \left(\sup _{n}\left(-a b \beta G^{1-b} S^{(b-1) \beta} n^{2-2 / b}+\left|\tilde{R}_{n}\right|\right)\right) .
\end{aligned}
$$

Let now $n \in \mathbf{N}$ be given and let $m \in \mathbf{N}$ be such that $m_{n}<m \leq m_{n+1}$, and consider $r_{m_{n}}^{m}$. We note by Lemma 3.2 and (3.14) that

$$
\log \left(r_{m_{n}}^{m}\right)=m \log \left(1-G S^{-\beta} n^{-2 / b}+O\left(n^{-4 / b}\right)\right) .
$$

Since $m \leq m_{n+1} \leq C n^{\alpha}=C n^{2+2 / b}$ and $2+2 / b-4 / b \leq 0$, we find using the Taylor expansion (3.3) that

$$
m \log \left(1-G S^{-\beta} n^{-2 / b}+O\left(n^{-4 / b}\right)\right)=m \log \left(1-G S^{-\beta} n^{-2 / b}\right)+O(1)
$$

which implies

$$
C_{1}\left(1-G S^{-\beta} n^{-2 / b}\right)^{m} \leq r_{m_{n}}^{m} \leq C_{2}\left(1-G S^{-\beta} n^{-2 / b}\right)^{m}
$$

for some constants $0<C_{1}<C_{2}$. Combining (2.3), (4.1), and (4.2) yields (2.7) of Theorem 2.2.

However, if $1<b \leq 2$, in (3.12), the exponent of $m_{n}^{(b-1) \beta}=S^{(b-1) \beta} n^{(b-1) \alpha \beta}=$ $S^{(b-1) \beta} n^{2-2 / b}$ is positive, although $\left|\tilde{R}_{n}\right|$ is bounded. Instead of (4.1) we use

$$
\begin{aligned}
& c_{1} \exp \left(-a G^{-b} S^{b \beta} n^{2}-\beta(a b)^{2 \beta} S^{(b-1) \beta} n^{2-2 / b}\right) \leq v\left(r_{m_{n}}\right) \\
& \leq c_{2} \exp \left(-a G^{-b} S^{b \beta} n^{2}-\beta(a b)^{2 \beta} S^{(b-1) \beta} n^{2-2 / b}\right),
\end{aligned}
$$

since $a b G^{1-b}=(a b)^{2 \beta}$. Lemma 3.2 yields for $r_{m_{n}}^{m}$

$$
\begin{aligned}
\log \left(r_{m_{n}}^{m}\right) & =m \log \left(1-G S^{-\beta} n^{-2 / b}+\beta G^{2} S^{-2 \beta} n^{-4 / b}+O\left(n^{-6 / b}\right)\right) \\
& =m\left(-G S^{-\beta} n^{-2 / b}+\left(\beta-\frac{1}{2}\right) G^{2} S^{-2 \beta} n^{-4 / b}+O\left(n^{-6 / b}\right)\right) \\
\Longrightarrow \log \left(r_{m_{n}}^{m}\right) & =m \log \left(1-G S^{-\beta} n^{-2 / b}+\beta G^{2} S^{-2 \beta} n^{-4 / b}\right)+O(1),
\end{aligned}
$$

since here $m n^{-6 / b} \leq m_{n+1} n^{-6 / b} \leq C n^{2+2 / b-6 / b} \leq C^{\prime}$. Hence, we have

$$
\begin{aligned}
& C_{1}\left(1-G S^{-\beta} n^{-2 / b}+\beta G^{2} S^{-2 \beta} n^{-4 / b}\right)^{m} \leq r_{m_{n}}^{m} \\
& \leq C_{2}\left(1-G S^{-\beta} n^{-2 / b}+\beta G^{2} S^{-2 \beta} n^{-4 / b}\right)^{m},
\end{aligned}
$$

and thus (2.8) follows.

Remark 4.1. It seems that the calculation of the numbers $m_{n}$ for the weights

$$
v(r)=\exp \left(-a /(1-r)^{b}\right)
$$

with our method becomes increasingly difficult for large $b$. Technical problems are caused by the fact that using an asymptotic expansion like (3.4) to evaluate $v\left(r_{m}\right)$, 
more terms are required, depending on how large $b$ is. Also, it seems that one would need a more complicated ansatz

$$
m_{n}=S_{\alpha} n^{\alpha}+S_{\alpha-1} n^{\alpha-1}+\ldots .
$$

The technical difficulties become obvious.

\section{The space of multipliers $\left(H_{v}^{\infty}(\mathrm{D}), \ell^{p}\right)$}

Let $A$ and $B$ be vector spaces of complex sequences containing the space of all the sequences with finitely many non-zero coordinates. The set of multipliers from $A$ into $B$ is

$$
(A, B):=\left\{c=\left(c_{n}\right):\left(c_{n} a_{n}\right) \in B \forall\left(a_{n}\right) \in A\right\} .
$$

Given a strictly increasing, unbounded sequence $J=\left(m_{n}\right)_{n=0}^{\infty} \subset \mathbf{N}$ and $1 \leq$ $p, q \leq \infty$ we denote as in [5, Definition 2],

$$
\ell^{J}(p, q):=\left\{\left(a_{m}\right)_{m=0}^{\infty}:\left(\sum_{m=m_{n}+1}^{m_{n+1}}\left|a_{m}\right|^{p}\right)^{1 / p} \in \ell_{q}\right\},
$$

with the obvious changes when $p$ or $q$ is $\infty$. The space $\ell^{J}(p, q)$ is a Banach space when endowed with the canonically defined norm. Observe that $\ell^{J}(p, p)=\ell_{p}$. We recall the following result from [5, Theorem 1.1] (see also [7, Lemma 5.1]).

Lemma 5.1. For $1 \leq p \leq \infty$ we have

$$
\left(\ell^{J}(2, \infty), \ell^{p}\right)=\ell^{J}(r, s)
$$

where (a) $r=2 p /(2-p), s=p$, if $1 \leq p<2$, (b) $r=\infty$, $s=p$, if $2 \leq p<\infty$, and (c) $r=s=\infty$, if $p=\infty$.

Proposition 5.2. Let $v(r)=\exp (-1 /(1-r)), r \in[0,1[$ and $1 \leq p \leq \infty$. Then, the space of multipliers $\left(H_{v}^{\infty}(\mathbf{D}), \ell_{p}\right)$ is the set of sequences $\left(\lambda_{m}\right)_{m=0}^{\infty}$ such that

$$
\left(\sum_{n=1}^{\infty}\left(\sum_{m=n^{4}+1}^{(n+1)^{4}}\left(\left|\lambda_{m}\right| e^{n^{2}}\left(1-\frac{1}{n^{2}}\right)^{-m}\right)^{\frac{2 p}{2-p}}\right)^{\frac{2-p}{2}}<\infty\right.
$$

if $1 \leq p<2$,

$$
\left(\sum_{n=1}^{\infty}\left(\max _{n^{4}<m \leq(n+1)^{4}}\left|\lambda_{m}\right| e^{n^{2}}\left(1-\frac{1}{n^{2}}\right)^{-m}\right)^{p}\right)^{\frac{1}{p}}<\infty,
$$

if $2 \leq p<\infty$, and

$$
\sup _{n \in \mathbf{N}}\left(\max _{n^{4}<m \leq(n+1)^{4}}\left|\lambda_{m}\right| e^{n^{2}}\left(1-\frac{1}{n^{2}}\right)^{-m}\right)<\infty,
$$

if $p=\infty$,

Proof. Since $\ell^{p}$ is a solid space, we have (cf. [1])

$$
\left(H_{v}^{\infty}(\mathbf{D}), \ell_{p}\right)=\left(S\left(H_{v}^{\infty}(\mathbf{D})\right), \ell_{p}\right) .
$$

Now, by Theorem 2.2 it is easy to see that $\left(\lambda_{m}\right)_{m=0}^{\infty} \in\left(S\left(H_{v}^{\infty}(\mathbf{D})\right), \ell_{p}\right)$, if and only if

$$
\left(\left(\left(e^{n^{2}}\left(1-\frac{1}{n^{2}}\right)^{-m}\right)\left|\lambda_{m}\right|\right)_{m=n^{4}+1}^{(n+1)^{4}}\right)_{n=0}^{\infty} \in\left(\ell^{J}(2, \infty), \ell_{p}\right) .
$$


The conclusion now follows from Lemma 5.1.

It is clear that Proposition 5.2 can be extended to more weights, but we prefer to present here only this more precise formulation as an example.

Acknowledgements. The research of Bonet was partially supported by the projects MTM2013-43540-P and MTM2016-76647-P. This paper was completed during the Bonet's stay at the Katholische Universität Eichstätt-Ingolstadt (Germany). The support of the Alexander von Humboldt Foundation is greatly appreciated. The research of Taskinen was partially supported by the Väisälä Foundation of the Finnish Academy of Sciences and Letters.

\section{References}

[1] Anderson, J. M., and A. L. Shields: Coefficient multipliers of Bloch functions. - Trans. Amer. Math. Soc. 224, 1976, 255-265.

[2] Bennet, G., D. A. Stegenga, and R. M. Timoney: Coefficient of Bloch and Lipschitz functions. - Illinois J. Math. 25, 1981, 520-531.

[3] Bierstedt, K. D., J. Bonet, and A. Galbis: Weighted spaces of holomorphic functions on bounded domains. - Michigan Math. J. 40, 1993, 271-297.

[4] Bierstedt, K. D., J. Bonet, and J. Taskinen: Associated weights and spaces of holomorphic functions. - Studia Math. 127, 1998, 137-168.

[5] Blasco, O., and C. Zaragoza-Benzosa: Multipliers on generalized mixed norm sequence spaces. - Abstr. Appl. Anal. 2014, 2014, Art. ID 983273.

[6] Bonet, J., P. Domański, M. Lindström, and J. Taskinen: Composition operators between weighted Banach spaces of analytic functions. - J. Aust. Math. Soc. Ser. A 64, 1998, 101-118.

[7] Bonet, J., and J. TASkinen: Solid hulls of weighted Banach spaces of entire functions. - Rev. Mat. Iberoam. (to appear).

[8] Constantin, O., and J. A. Peláez: Boundedness of the Bergman projection on $L_{p^{-}}$spaces with exponential weights. - Bull. Sci. Math. 139:3, 2015, 245-268.

[9] Dostanić, M. R.: Multipliers in the space of analytic functions with exponential mean growth. - Asymptot. Anal. 65:3-4, 2009, 191-201.

[10] Dostanić, M. R.: Integration operators on Bergman spaces with exponential weight. - Rev. Mat. Iberoam. 23:2, 2007, 421-436.

[11] Jevtić, M., and M. Pavlović: On the solid hull of the Hardy-Lorentz space. - Publ. Inst. Math. (Beograd) (N.S.) 85:99, 2009, 55-61.

[12] Jevtić, M., D. Vukotić, and M. Arsenović: Taylor coefficients and coefficient multipliers of Hardy and Bergman-type spaces. - RSME Springer Series 2, Springer, 2016.

[13] Lusky, W.: On the isomorphism classes of weighted spaces of harmonic and holomorphic functions. - Studia Math. 175:1, 2006, 19-40.

[14] PAu, J., and J. A. Peláez: Volterra type operators on Bergman spaces with exponential weights. - In: Topics in complex analysis and operator theory, Contemp. Math. 561, Amer. Math. Soc., Providence, RI, 2012, 239-252.

[15] Pavlović, M.: On harmonic conjugates with exponential mean growth. - Czechoslovak Math. J. 49, 1999, 733-742.

[16] Pavlović, M.: Function classes on the unit disc. An introduction. - de Gruyter Stud. Math. 52, de Gruyter, Berlin, 2014.

[17] PelÁez, J.A., and J. RÄTtyä: Weighted Bergman spaces induced by rapidly increasing weights. - Mem. Amer. Math. Soc. 227:1066, 2014.

[18] Shields, A. L., and D. L. Williams: Bounded projections, duality and multipliers in spaces of analytic functions. - Trans. Amer. Math. Soc. 162, 1971, 287-302. 